LIAMES, Campinas, SP, v. 21, 1-18, e021007, 2021

\title{
A distinção massa e contável na gramática Rikbaktsa (Macro-Jê) ${ }^{1}$
}

\author{
Érica Milani Dellai \\ Universidade Federal de Santa Catarina, Brasil \\ https://orcid.org/0000-0003-4773-5509 \\ Vitória Maria Jasper Ern \\ Universidade Federal de Santa Catarina, Brasil \\ https://orcid.org/0000-0002-1062-3530 \\ Léia de Jesus Silva \\ Universidade Federal de Goiás, Brasil \\ Universidade Federal de Santa Catarina, Brasil \\ https://orcid.org/0000-0002-8835-377X \\ Roberta Pires de Oliveira \\ Universidade Federal de Santa Catarina, Brasil \\ Universidade Federal do Paraná, Brasil \\ Conselho Nacional de Desenvolvimento Científico e Tecnológico, Brasil \\ https://orcid.org/0000-0002-4946-7205 \\ Beatriz Martins Rachadel \\ Universidade Federal de Santa Catarina, Brasil \\ https://orcid.org/0000-0001-5520-8704 \\ Bianca Maria de Souza \\ Universidade Federal de Santa Catarina, Brasil \\ https://orcid.org/0000-0002-4876-6983
}

\begin{abstract}
The paper investigates the count-mass distinction in Rikbaktsa (Macro-Jê). It presents the results of data collected following Lima and Rothstein's questionnaire (2020). The data was gathered using an unpublished corpus, the scarce literature on this language (Boswood 1971, 1978; SIL 2007; Silva 2011), and elicitation with a native speaker. The analysis shows that there is plural morphology which does not combine with mass nouns. Nouns that denote stable atoms combine directly with numerals. Substance nouns require measure phrases to be counted. These are indications that this language can be classified as a number marking language (Chierchia 2010, 2015), even if there are some mass nouns that combine directly with numerals. None of the fifteen languages in Lima \& Rothstein (2020) are reported to have a specialized mass morpheme. Chacon (2012) claims that Kubeo (Tukano-Oriental) has a demonstrative for mass. This is also the case in Rikbaktsa, but in Rikbaktsa, it is spread across the grammar: the demonstrative $n a$ 'this' combines with a third person marker on the personal pronoun $a$ $n a$, with interrogative proforms, and in attributive constructions. This study contributes to a better understanding of count-mass distinction across languages and of the grammar of this minority language.

KEYWORDS: Count-mass distinction; Demonstratives; Rikbaktsa
\end{abstract}

RESUMO: Neste artigo investigamos a distinção massivo-contável na língua Rikbaktsa (Macro-Jê). Partimos do questionário de Lima \& Rothstein (2020), o qual procuramos responder levantando os dados em um acervo inédito do qual já dispúnhamos, na pouca literatura sobre a língua (Boswood 1971, 1978; SIL 2007; Silva 2011), e em

\footnotetext{
${ }^{1}$ Agradecemos aos pareceristas que avaliaram uma versão preliminar deste artigo. Os erros são de nossa responsabilidade.
} 
uma elicitação de dados com um falante nativo. A análise mostrou que há morfologia de plural e que ela não se combina com nomes de massa. Nomes que denotam átomos estáveis se combinam diretamente com numerais. Para serem contados, nomes de substância exigem a presença de sintagmas de medidas. Esses são indícios de que a língua em questão pode ser caracterizada como uma língua de número marcado (Chierchia 2010, 2015), mesmo que haja alguns nomes de massa que podem ser contados diretamente. Em nenhuma das quinze línguas apresentadas em Lima \& Rothstein (2020) há relato de um morfema especializado para marcar massa. Chacon (2012) afirma que o Kubeo (Tukano-Oriental) exibe um demonstrativo de massa. Esse é também o caso em Rikbaktsa, mas nesta língua o demonstrativo $n a$ 'isto', que seleciona nomes massivos, se espalha pela gramática: aparece com marca de terceira pessoa no pronome pessoal $a$-na, com proformas interrogativas e em construção atributiva. Este estudo contribui, portanto, para uma melhor compreensão da distinção massivo-contável através das línguas e da gramática desta língua minoritária.

Palavras-CHaVes: Distinção massivo-contável; Demonstrativos; Rikbaktsa

\section{Notas preliminares}

A língua Rikbaktsa (ISO 639-3: rkb) ${ }^{2}$ é a única da família Rikbaktsa e pertence ao tronco linguístico Macro-Jê (Boswood 1971: 49; Rodrigues 1986). Os Rikbaktsa são hoje aproximadamente 1.600 pessoas (Áthila 2019: 718) vivendo em três terras indígenas localizadas no noroeste do estado do Mato Grosso (Brasil). Silva (2011: 23) descreve o uso da língua por diferentes grupos etários. Entre crianças e jovens, predomina o monolinguismo em português com eventuais exceções bilíngues. Entre os adultos, há mais variação: alguns são bilíngues, outros têm uma boa compreensão do Rikbaktsa, mas não falam esta língua; entre as pessoas com menos de 30 anos predomina o monolinguismo em português. Entre idosos, a autora afirma que há indivíduos bilíngues, bem como monolíngues em Rikbaktsa.

O processo de substituição da língua Rikbaktsa pelo português brasileiro teve início em meados do século XX, decorrente do contato entre a sociedade envolvente e esse povo, iniciado a partir da invasão seringueira em suas terras e de processos de pacificação subsequentes (Pacini, 1999). O impacto gerado na educação formal de crianças e jovens do fim do século passado fez com que, embora compreendessem a língua Rikbaktsa, evitassem utilizá-la, dificultando a transmissão da língua para as gerações mais novas (Áthila 2006: 59). Está em curso um processo de vitalização da língua, atualmente ensinada nas escolas como segunda língua.

Neste artigo discutimos a distinção massa e contável nessa língua, tendo como pano de fundo o panorama atual na Linguística (Chierchia 2010, no prelo; Rothstein 2010, no prelo; inter alia). $\mathrm{Na}$ seção 2, apresentamos o quadro teórico usado. Buscamos preencher o questionário de Lima \& Rothstein (2020) em Rikbaktsa com base em dados de que já dispúnhamos, e dados encontrados na literatura sobre a língua, ${ }^{3}$ a fim de poder comparar essa língua às demais línguas indígenas ali descritas. Discutimos a metodologia de investigação na seção 3. Na seção 4, mostramos os resultados da nossa pesquisa: o Rikbaktsa não apenas distingue massa e contável através da combinação com a morfologia de número, mas exibe um morfema que seleciona nomes de massa. Nenhuma das quinze línguas descritas em Lima \& Rothstein (2020) apresenta um morfema exclusivo para massa, mas Chacon (2012) descreve o fenômeno em Kubeo (Tukano-Oriental). A conclusão aponta caminhos de investigação e desdobramentos práticos.

Propomos aqui um tratamento semântico para a questão da distinção massivo-contável em Rikbaktsa, o que nos permitirá sustentar a hipótese de que essa é uma língua de número marcado no sintagma nominal, como é o caso do inglês e do português brasileiro, mas

\footnotetext{
${ }^{2}$ https://iso639-3.sil.org/code/rkb

${ }_{3}^{3}$ Considerando o contexto pandêmico, não foi possível trabalhar com o questionário de Lima e Rothstein (2010) em campo, junto a falantes nativos.
} 
diferentemente destas línguas, exibe morfologia para massa obrigatória no determinante, um tema a ser explorado.

\section{A distinção massa e contável na Linguística contemporânea}

Compartilhamos com outras espécies a capacidade de distinguir substâncias e objetos (Soja et al. 1991; inter alia), entretanto as línguas variam quanto a essa distinção. Para compreender o panorama atual na semântica é preciso ter em mente a diferença entre objetos e substâncias no mundo e as palavras e sua gramática. Os mesmos objetos no mundo podem ser categorizados diferentemente pelas línguas, não havendo, portanto, uma correspondência direta entre objetos no mundo e ser contável ou massivo. Isto pode ser observado, por exemplo, com o objeto cabelo que em italiano (capeli) é contável, ao passo que em inglês (hair) é massivo. Em Rikbaktsa, segundo Silva (2011: 42), os nomes abaixo, por exemplo, são massivos, porque, como veremos na próxima seção, não se combinam com morfologia de plural, enquanto suas contrapartes, em português (e também em inglês), são contáveis:

$\begin{array}{ll}\text { rodo } & \text { 'batata' } \\ \text { pitfi } & \text { 'castanha-do-pará' } \\ \text { tumado } & \text { 'banana' } \\ \text { wahoro } & \text { 'casa' }\end{array}$

Além de haver variação entre as línguas, uma mesma língua pode ter nomes diferentes para o mesmo objeto no mundo. Por exemplo, shoes e footwear denotam o mesmo conjunto de objetos no mundo, no entanto shoes é contável e footwear é massivo. Há, ainda, mecanismos na própria língua que permitem converter massa em contável e vice-versa. Duas cervejas é um caso de mudança de massa para contável, enquanto em tem batata na salada, batata é compreendida massivamente (Pelletier 1975; Chierchia 2010; Rothstein 2010). Esses mecanismos de mudança são conhecidos pela literatura e sabemos que eles são restritos; por isso, a hipótese é de que haja coerção nesses casos. A transformação de massa em contável exige recipientes que são conhecimento compartilhado, enquanto a transformação de contável em massivo ocorre em contextos de comida (Frazier et al. 2005; inter alia).

Nem todos os nomes massivos são substância, alguns nomeiam objetos no mundo, como é o caso dos nomes de massa objeto (Rothstein 2017) ou nomes falsos de massa (Chierchia 2010), como furniture 'mobília' no inglês. Segundo Chierchia (2010), esse tipo de nome só ocorre em línguas marcadas para número, como o inglês. Esses nomes, em tarefas de julgamento de quantidade, são contados e não medidos (Barner \& Snedeker 2005). Além disso, Rothstein (2010) mostra que há nomes contáveis que não denotam unidades atômicas no mundo, como é o caso de reta, cerca, buquê, e propõe uma operação de atomização (Rothstein 2010, 2017). Há ainda os chamados nomes flexíveis, que ora se comportam como contável ora como massa: stone 'pedra' e stones 'pedras' em inglês, por exemplo. Essas constatações levaram autores como Pelletier $(1975,2009,2012)$ a afirmar que todos os nomes são potencialmente massivos e contáveis, e caberia à sintaxe fazer a distinção.

A diferença clássica entre nomes de objeto e nomes de massa se ancora na possibilidade ou não de se combinar diretamente com numerais. No entanto, os critérios para definir se um nome é contável ou massivo também se mostraram problemáticos quando comparamos as línguas. A pesquisa empírica mostrou, por exemplo, que há línguas que não se comportam como previsto pela literatura, quer porque tratam massivamente nomes contáveis - como parece ser o caso do Singular Nu no português brasileiro (Pires de Oliveira \& Rothstein 2011; Rothstein \& Pires de Oliveira 2020) - , quer porque contam diretamente massa - como ocorre 
no Yudja ${ }^{4}$ (Lima 2010, 2014). A dimensão da problemática pode ser medida pelo fato de que só recentemente foram publicados dois livros sobre a questão: Moltmann (2020) e Kiss et al. (no prelo). Além do volume de 2020, Linguistic Variation, editado por Lima \& Rothstein (2020), que trata da questão a partir da aplicação de um questionário sobre massa e contável em algumas línguas indígenas brasileiras. Este questionário motivou a pesquisa apresentada neste artigo.

Para explicar a variação entre as línguas, Chierchia (2010, 2015, no prelo) propõe o parâmetro do número, que pode ser compreendido como um roteiro para investigarmos as línguas. Esse é o caso do questionário em Lima \& Rothstein (2020), que tem como pano de fundo os parâmetros semânticos (Chierchia 1998, 2010). O parâmetro semântico em Chierchia (2010, 2015) distingue três tipos de língua com base no modo como as línguas combinam nomes com numerais: línguas de número marcado no nome, classificadoras e de número neutro. A hipótese é que todas as línguas distinguem massa e contável na gramática, embora não necessariamente da mesma forma.

As línguas de número marcado no nome, como o inglês, se caracterizam pela obrigatoriedade da morfologia de número no nome e pelo fato de que só nomes contáveis se combinam diretamente com numerais:

$$
\begin{aligned}
& \text { Three cats } \\
& \text { 'Três gatos' }
\end{aligned}
$$

\section{*Three waters \\ '*Três águas'}

O numeral é um modificador do nome que seleciona predicados atômicos (Chierchia 2015: 18). Um predicado atômico denota um conjunto de indivíduos que são átomos estáveis, isto é, a unidade se mantém a mesma através das alternativas epistêmicas. Nomes de massa denotam átomos instáveis, ou seja, sabemos que eles podem se alterar ao longo dos mundos, e por isso, eles não se combinam com morfologia de plural, nem com numerais. Os falantes entendem que gatos denotam unidades estáveis de gato, enquanto não há uma única maneira de detectar uma unidade de água e, por isso, não é possível contar.

Assim, nas línguas de número marcado, os nomes de massa não se pluralizam, o sistema de determinantes é sensível à distinção massa e contável, e existem falsos massivos, caso de furniture. Estes últimos são nomes que se comportam gramaticalmente como massivos (não permitem flexão de número e não se combinam com numerais diretamente), embora denotem objetos no mundo e sejam contados pelos falantes quando estes são chamados a participar em testes de julgamento de quantidade com perguntas como Who has more furniture? 'quem tem mais mobília' (Barner \& Snedeker 2005).

As línguas do tipo classificadoras, por sua vez, não têm morfologia de número e nelas nenhum nome se combina diretamente com numerais. Para que essa combinação seja possível, é preciso a mediação de um classificador que, segundo Chierchia, transforma o indivíduoespécie em um predicado de indivíduos (veja a derivação semântica em Chierchia 2010: 149). Assim, ge no exemplo em (4) do Mandarim transforma a espécie que é denotada pelo nome, nanhai, 'menino', no predicado, permitindo então a combinação com o numeral.

(4) san *(ge) nanhai

três CL menino

'três meninos'

(Chierchia 2010: 107 - exemplo 15a)

\footnotetext{
4 A língua Yudja é classificada entre as línguas da família Juruna, tronco linguístico Tupi. https://pib.socioambiental.org/pt/Povo:Yudj\%C3\%A1/Juruna
} 
Os classificadores indicam a distinção massa e contável: a aceitabilidade da combinação de ge com nomes massivos, como em (5), é marcada.

$$
\begin{array}{ll}
\text { ??san ge } & \text { xue } \\
\text { três CL } & \text { sangue } \\
\text { 'três porções de sangue' }
\end{array}
$$

A hipótese do parâmetro é que as línguas diferem na primeira bifurcação que estrutura uma raiz em nome, $n$-inho (little $\mathrm{n}$ ) na terminologia da morfologia distribuída. O inglês inicia com predicados de indivíduos, enquanto que o Mandarim parte dos indivíduos espécie. Assim, nesta língua, a combinação com o classificador é obrigatória porque ele muda o tipo semântico de espécie para um predicado de indivíduo. E somente desta forma é possível a combinação com o numeral.

Nesta proposta, classificadores não se confundem com sintagmas de medidas, os quais ocorrem com nomes de massa no inglês e no português, exemplificado em 3 glasses of wine ' 3 cálices de vinho'. Mais uma vez, sem entrar em maiores detalhes, ${ }^{5}$ podemos ter uma leitura em que contamos os cálices, e nesse caso of wine é um modificador, e uma leitura onde o que importa é a medida. Assim, podemos ter uma jarra com a medida equivalente a três cálices. Neste caso, glasses é um medidor e o núcleo é wine.

Por fim, Chierchia (2010) delimita o grupo de línguas denominadas de número neutro, que não marcam a pluralidade obrigatoriamente no nome e tampouco possuem classificadores obrigatórios. Os nomes sem marcação de plural podem apresentar leituras tanto de singular quanto de plural. Os nomes contáveis podem combinar-se diretamente com numerais, sem a necessidade de um classificador. Assim como no inglês, os nomes de massa exigem um sintagma de medida para se associar com numerais, ${ }^{6}$ como podemos perceber nos dados do Dëne Sułine (Língua Atapascana):

$$
\begin{aligned}
& \text { *solaghe bër } \\
& \text { cinco carne } \\
& \text { 'cinco carne(s)' }
\end{aligned}
$$$$
\text { (Wilhelm 2008: } 47 \text { - exemplo 9b) }
$$

$$
\begin{array}{lll}
\text { solaghe } & \text { nedadhi } & \text { bër } \\
\text { cinco } & \text { libra } & \text { carne } \\
\text { 'cinco libras (de) carne' } &
\end{array}
$$$$
\text { (Wilhelm 2008: } 47 \text { - exemplo 10a) }
$$

Tendo como pano de fundo a hipótese dos parâmetros semânticos teorizados por Chierchia $(1998,2010)$ e a discussão na literatura sobre massa e contável (Rothstein 2017, entre outros), o questionário apresentado em Lima \& Rothstein (2020) se propõe a observar o comportamento dos numerais, plurais, quantificadores, classificadores, sintagmas de recipiente/medida e comparativos em línguas indígenas brasileiras. Para as autoras, este questionário procura servir como um guia para a pesquisa e descrição da distinção massacontável em línguas sub-representadas, permitindo assim, traçar um caminho de análise comparativa entre elas.

Um dos efeitos da hipótese dos parâmetros foi disparar uma onda de investigações em línguas pouco conhecidas, como é o caso do Dëne Sułiné. buscando verificar a proposta e as afirmações encontradas em Chierchia (1998). Talvez a afirmação mais questionada tenha sido

\footnotetext{
${ }^{5}$ Ver Rothstein (no prelo) para uma análise detalhada dessa construção e sua ambiguidade.

${ }^{6}$ Wilhelm (2008: 48) afirma que "No Dëne Sułine (Língua Atapascana), termos de medida são opcionais para alguns nomes compatíveis com numerais (como tthe 'stone', $k e$ 'shoe') e eles são requeridos por outros nomes (como lígofi 'café', ji'etué 'vinho', suga 'açúcar')".
} 
o que Chierchia chama de "assinatura do nome de massa": segundo o autor, estes nomes não se combinam diretamente com numerais. Lima (2014), entre outros, mostra que em Yudja (Juruna) isso parece ocorrer, desafiando, assim, a propriedade assinatura de massa. Abaixo está um exemplo da autora discutido em Chierchia (2015: 24). No contexto, alguém cortou o dedo e caiu uma gota de sangue perto da escola, uma outra perto do hospital e uma perto do rio (as gotas de sangue têm tamanhos e formatos diferentes):

$$
\begin{aligned}
& \text { txabüu apeta } \quad \text { pepepe } \\
& \text { três sangue cair.REDUPL (três eventos) } \\
& \text { 'três (gotas de) sangue caíram no chão' }
\end{aligned}
$$$$
\text { (Lima 2014: } 58 \text { - exemplo 28b) }
$$

O numeral 3, txabïu, se combina diretamente com um nome de massa, apeta ('sangue'). Esse tipo de dado, que ocorre em várias das línguas descritas em Lima \& Rothstein (2020), leva as autoras a desafiar a generalização de que a contagem direta se restringe a nomes contáveis. No entanto, Chierchia (no prelo) apresenta uma derivação para o sintagma em Yudja, que teria um sistema de classificadores encobertos. Assim, para ele, esta é uma língua classificadora, como o Mandarim. Essa discussão provê as lentes através das quais investigamos a distinção massa e contável em Rikbaktsa.

\section{Metodologia}

O objetivo era entender a distinção massivo-contável em Rikbaktsa tendo como guia o questionário de Lima \& Rothstein (2020), visando poder comparar essa língua às demais descritas no volume em questão. O corpus da nossa análise é composto por dados encontrados na literatura sobre a gramática Rikbaktsa (Boswood 1971, 1978; Silva 2011), pelo Dicionário Bilíngue (SIL 2007), por dados inéditos do acervo pessoal de Silva e por uma única coleta, realizada remotamente, com um professor Rikbaktsa. ${ }^{7}$

Uma vez que o Dicionário Bilíngue (SIL 2007) e Boswood (1971, 1978) não apresentam glosas e estão escritos na ortografia proposta para a língua, como exemplificado em (9a), acrescentamos a estes dados duas linhas: uma, em escrita fonético-fonológica, seguindo a proposta de Silva (2011), e outra com glosas, mantendo assim a escrita e a tradução dos autores, como demonstrado em (9b):

\section{a. Parini pazaharetsa petoktsa nisiboro.}

'A onça comeu dois porcos'.

(SIL 2007: 26)

b. Parini pazaharetsa petoktsa nisiboro parini parahei-tfa petok-tfa ni- $\phi$-ji-boro onça porco-NF.PL dois-NF.PL 3SUJ-PAS-3OBJ.PL-comer 'A onça comeu dois porcos'

(SIL 2007: 26)

Os dados que pertencem ao acervo pessoal de Silva, ainda não publicados, foram coletados em viagens anuais a duas terras indígenas Rikbaktsa (Erikpaktsa e Japuira, no Mato Grosso) entre os anos de 2003 e 2010 . Nestas ocasiões, por meio de entrevistas com falantes nativos, a autora registrou textos narrativos; listas de palavras; sentenças e depoimentos, solicitados e espontâneos. ${ }^{8}$

\footnotetext{
7 O falante tem 45 anos e é bilíngue, tendo o Rikbaktsa como L1.

${ }^{8}$ Os dados cujas referências não foram indicadas correspondem aos dados inéditos do acervo pessoal de Silva.
} 


\section{A gramática e a questão massa e contável em Rikbaktsa}

Como em várias outras línguas, em Rikbaktsa não há artigos definidos nem indefinidos, ou seja, os nomes aparecem nus quando em posição argumental. Em (10) vemos a estrutura básica de uma sentença não marcada: os nomes nus estão em posição à esquerda e são retomados anaforicamente pelos índices pronominais atachados aos verbos, $n i$ - para o sujeito e - $f i$ para o objeto plural. Silva (2011) nota que nessa língua a estrutura verbal é uma sentença, expressando uma proposição.

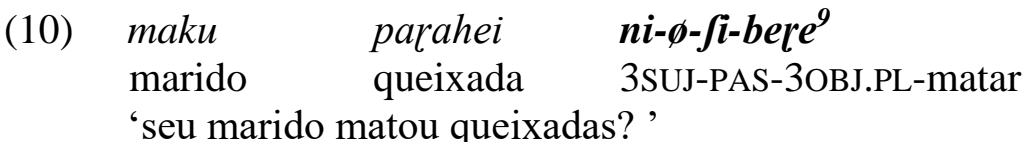

(Silva 2011: 386)

O nome parahei 'queixada' não carrega marca de plural. No entanto, sabemos que sua interpretação é plural porque ele é retomado pelo índice pronominal plural $-f i$ '3OBJ.PL', atachado ao verbo. Em outros termos, embora muito produtiva (como exemplificado em (11), abaixo), a marcação de plural não é obrigatória no nome.

\section{(11) (maku-tfa) \\ homem-NF.PL \\ (taha-boto-tfa) \\ 3COR.PL-flecha-NF.PL}

$n i-\phi-\int i-\int a r a-k a=n a h a$

3SUJ-PAS-3OBJ.PL-fazer.flecha-CONT=PL.SUJ

'(os homens) eles estavam fazendo-as (as flechas deles)'

(Silva 2011: 30)

Independentemente de se tratar de uma sentença interrogativa, como em (10), ou uma declarativa como em (11), a ordem não marcada dos constituintes na sentença é SOV. Em (11) observamos ainda que o plural nu maku-tfa 'homens' precede o objeto, também um plural nu, taha-boto-tfa 'flechas', e ambos são retomados por índices pessoais na estrutura verbal na mesma ordem: o sujeito $n i$ - '3SUJ', depois o objeto $-f i$ '3OBJ.PL'. No verbo, o plural do sujeito é marcado pelo clítico naha. Os constituintes nominais são opcionais.

A partir do pouco que foi mostrado acima, entende-se que os sintagmas nominais são nus e a ausência de marca de plural não é indicação de singularidade, pois como vimos em (10), o plural pode aparecer apenas no índice pessoal marcado sobre o verbo. Essa é uma propriedade importante para entendermos a marcação de número e a questão massa e contável nesta língua. Apresentamos, a seguir, os resultados da nossa investigação feita a partir do questionário de Lima \& Rothstein (2020).

\subsection{Os nomes e o morfema de plural}

A primeira seção do questionário de Lima \& Rothstein (2020) é um levantamento de léxico já com o propósito de verificar se a língua tem uma gama de tipos de nomes: nomes para objetos e substâncias; nomes para grãos; nomes coletivos ou agregados, como instrumento; e nomes de massa objeto, como furniture. Após esse levantamento de léxico, a tarefa é verificar se a língua distingue morfologicamente nomes contáveis de nomes massivos.

O Rikbaktsa é uma língua que marca morfologicamente o plural nos nomes, através de três sufixos: $-t f a,-r a,-k V(C)$, em que $\mathrm{V}$ corresponde à cópia da vogal imediatamente

\footnotetext{
${ }^{9}$ Interessante notar que o verbo também está na sua forma singularizada. O verbo correspondente a matar distingue o número do paciente lexicalmente, a forma bere indica matar um, enquanto a forma $b a$, matar mais de um.
} 
precedente e $\mathrm{C}$, à coda da última sílaba da raiz (Silva 2011: 74,79). A primeira marca de plural é utilizada para nomes que denotam coisas e entidades que não são fêmeas humanas. Já -ra e $k V(C)$ são usadas exclusivamente com nomes que denotam referentes humanos e fêmeas, sendo a última forma menos produtiva. Há ainda uma pequena subclasse de nomes de fêmeas que marcam plural por composição, combinados com o lexema kikitri $(\sim k i k i)$ 'mulheres', como em -tjik-kikiri, 'filhas' e mek-kikiri, 'outras'. Para os nossos propósitos interessa apenas que há morfologia de número.

Os exemplos em (12) mostram a distinção que ocorre, no plural, entre nomes de fêmeas e não fêmeas: jabui 'criança' combinada com o morfema -t fa significa 'meninos' ou 'crianças' (12a), mas associada ao morfema - $r a$ significa exclusivamente 'meninas' (12b):
a. jabui-t/a
criança-F.PL 'meninos/crianças'
(Silva 2011: 76)
b. jabui-ra criança-NF.PL 'meninas'
(Silva 2011: 77)

A flexão de plural, em qualquer uma de suas manifestações linguísticas, só se combina com nomes que denotam objetos no mundo, unidades que consideramos estáveis através das situações. Assim, nomes como maku 'homem', parahei 'porco/queixada' e moko 'mandioca' são pluralizados pelo sufixo - t fa. Essa é uma propriedade que caracteriza os nomes contáveis, i.e. eles se combinam com morfologia de plural. Enquanto nomes de substância como pihik 'água' e imirí 'areia' não aceitam nenhuma das marcas de plural, sendo, portanto, considerados massivos.

Como já vimos na seção teórica, os nomes apresentados em (1) são casos de não correspondência entre o português e o Rikbaktsa. Silva (2011: 42) destaca que pitfi 'castanha do Pará', tumado 'banana', wahoro 'casa', e rodo 'batata' são considerados massivos em Rikbaktsa já que, segundo a autora, construções como * rodo-tfa não são aceitas pelos falantes. Um segundo exemplo interessante dessa falta de correspondência entre as categorias destas línguas é o termo para arroz, exemplificado em (13), que, apesar de ser empréstimo do português, em Rikbaktsa é um nome contável e se combina, portanto, com morfologia de plural.

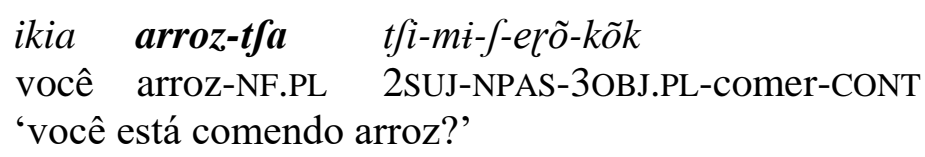

Procuramos em nosso corpus nomes falsos de massa (Chierchia 2010) ou nomes de massa objeto (Rothstein 2017), porque, segundo Chierchia, essa é uma propriedade exclusiva das línguas marcadas para número. Como já dissemos na seção teórica, esses são nomes que se comportam gramaticalmente como massa e denotam objetos no mundo (furniture é o exemplo prototípico). O dado a seguir corrobora a hipótese de que há nomes de massa objeto em Rikbaktsa:

\begin{tabular}{|c|c|c|c|c|}
\hline katse & bo & kanamyty & nyny & $i k y$ \\
\hline$k a-t \int e$ & bo & $k a-n a m \dot{t}=t \dot{t}$ & nini & $i k \dot{k}$ \\
\hline 1POSS.SG-filho & ALAT & 1POSS.SG-coisas=ACUS & dar & AUX.PAS \\
\hline
\end{tabular}


Embora na glosa em português apareça 'coisas' no plural, em Rikbaktsa este nome não tem nenhuma morfologia de plural e, segundo Silva, os falantes não aceitam combinar kanami 'meus pertences'/'minhas coisas' com o sufixo de plural, *kanamit fa, tal como observado com os nomes massivos. Além disso, kanami denota todos os pertences do falante, o que pode incluir rede, canoa, flecha, etc. Esse caso não se confunde com os exemplos em (1) porque nomes de massa objeto denotam objetos que são diferentes, a cadeira e o sofá para mobília por exemplo, enquanto os nomes em (1) denotam objetos que são do mesmo tipo, por assim dizer.

No corpus não encontramos nomes de agregados como artefatos, vegetais e mobília, nem termos genéricos para instrumento musical ou armamento. Encontramos, no entanto, um caso que parece ser de nome flexível, isto é, um nome que se comporta ora como massa, ora como contável, como é o caso de stone e stones em inglês. Trata-se do nome naha 'ornamentária/joia'. Discutiremos esse exemplo na seção 4.2, porque é sua combinação com a marca de massivo, tema da seção, e com a marca de plural que sustenta essa hipótese.

A investigação sobre o Rikbaktsa, a partir das primeiras seções do questionário de Lima \& Rothstein (2020), mostrou, como já adiantamos, que essa língua exibe morfologia de plural e distingue nomes contáveis de nomes massivos. Além disso, parece haver falsos nomes de massa/nomes de massa objeto e nomes flexíveis. Essas propriedades se conformam às predições universais de Chierchia (2010): substâncias correspondem a nomes de massa e não aceitam plural. Há ainda elasticidade, isto é, nomes flexíveis, que são ambíguos entre massa e contável. Esta língua apresenta também as características de línguas que marcam o número no sintagma nominal, como o inglês e o português, já que o morfema de plural se agrega ao nome e distingue os nomes contáveis dos massivos. Uma outra evidência que dá suporte a essa hipótese é a existência de falsos nomes de massa.

\subsection{Os numerais e a contagem}

Como vimos na seção 2, os numerais têm um papel fundamental para compreendermos a gramática de uma língua, pois eles permitem determinar se estamos diante de uma língua classificadora, em que nenhum nome é contado diretamente. Lima \& Rothstein (2020) argumentam que há línguas que são mais flexíveis quanto à possibilidade de contar massa diretamente.

Em Rikbaktsa, Boswood (1978: 68) apresenta numerais até 20, enquanto Polegatti (2013), até 10. No nosso corpus, entretanto, identificamos apenas ftuba 'um', em (15), e petok 'dois', em (16). O primeiro aparecendo mais que o segundo. Notamos que há uma preferência pelo uso do termo sizybarẽtsa [/i-ruba-ĩ-tfa 3PL-ser.muito-EST.AFIR-NF.PL] 'muitos' para quantidades a partir de três. ${ }^{10}$ Os exemplos (15) e (16) mostram que os numerais se combinam diretamente com nomes contáveis.

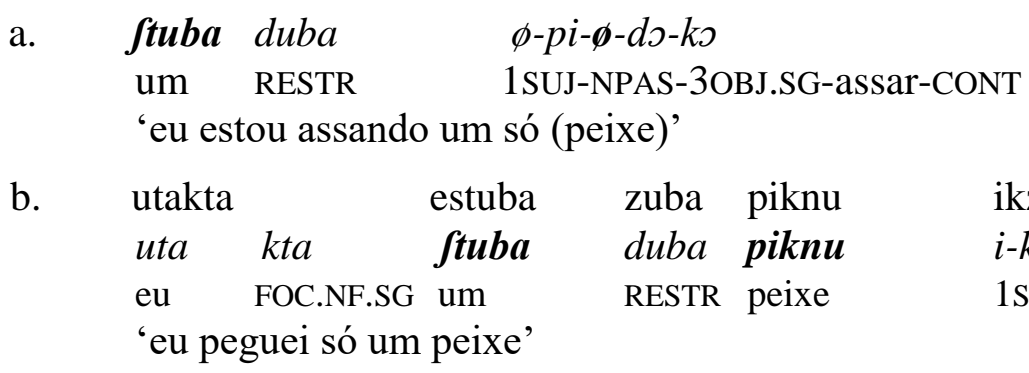

ikzizo. $i-k-i-d o$ 1SUJ-PAS-3OBJ.SG-pescar ${ }^{11}$

(SIL 2007: 11)

\footnotetext{
${ }^{10}$ Boswood (1978: 68) afirma que “às vezes se emprega ‘um' ou 'dois' para qualquer número pequeno, e para uma quantidade maior só 'muitos'. Às vezes também mostram os números nas mãos ou nos pés”.

${ }^{11}$ Há mais de uma série de morfemas para marcar a pessoa do sujeito, como se pode ver em (15a) e (15b). Para mais informações, consultar Silva (2011).
} 
(16)
a. abaka bato petok duba fi-fukra=bui=ra
agora não dois RESTR 3PL-ser.solteiro=NEG=F.PL
'agora, apenas duas não têm marido'
b. Parini pazaharetsa petoktsa nisiboro

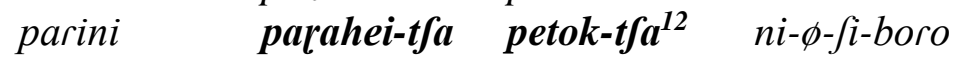
onça porco-NF.PL dois-NF.PL 3SUJ-PAS-3OBJ.PL-comer
'A onça comeu dois porcos'

(SIL 2007: 26)

\section{c. petok-ra hiri-ra \\ dois-F.PL filh(o/a)-F.PL \\ 'duas filhas (gêmeas)'}

Encontramos um único dado em que temos um numeral $\mathrm{cm}$ um nome de massa, em (17). O nome pitfi 'castanha', que como vimos em (1), não aceita morfologia de número, se combina diretamente com o numeral ftuba 'um':
ikra pitji
Stuba t/ik
$\phi-m \dot{t}$
eu.F castanha um quebrar
$1 \mathrm{SUJ}-\mathrm{NPAS}+\mathrm{AUX}$

'eu vou quebrar uma castanha (semente)'

(Silva 2011: 171)

Por ser este o único dado de que dispúnhamos, realizamos, virtualmente, uma coleta controlada com um falante bilíngue. O objetivo era entender se o Rikbaktsa é uma língua como o Yudja (Tupi) (Lima 2014) em que todos os nomes, contáveis e massivos, podem ser combinados diretamente com numerais; ou se se comporta como o Sakurabiat (Tupi) (Galucio \& Costa 2020 apud Lima \& Rothstein 2020: 195), em que alguns nomes de massa são diretamente combinados com numerais. Solicitado a avaliar as combinações abaixo, o falante aceitou contar rodo 'batata' diretamente, como mostram os dados em (18) a (20), mas rejeitou a combinação com o plural, em (21):
(18) Jtuba rodo
'uma batata'
(19) petok rodo
'duas batata'
(20) mitsihiwa rodo
'cinco batata'
$\begin{array}{ll}\text { (21) petok } & \text { rodo-tfa } \\ \text { dois } & \text { batata-NF.PL }\end{array}$

No entanto, o mesmo falante, rejeitou a combinação direta de numerais com nomes de substância, sendo consideradas agramaticais: *petok pihik 'duas água' e *petok tumì 'duas chicha'. Nesses casos, o falante utilizou o sintagma de medida fuk 'panela', como mostram os dados abaixo, fornecidos espontaneamente pelo falante ao corrigir * petok tumi 'duas chicha':

\footnotetext{
${ }^{12}$ A presença da flexão de plural no numeral dois parece ter uma relação com a categoria gramatical a qual este termo pertence. Entretanto, não nos deteremos nesse ponto, pois ele não parece interferir na compreensão global do fenômeno aqui investigado.
} 
(22)

Jtuba fuk tumi
uma panela chicha
'uma panela de chicha'

petok fuk tumi
duas panela chicha
'duas panelas de chicha'

Esses dados indicam que os nomes de substâncias exigem a presença de um sintagma de medida, sendo agramatical combinar o numeral diretamente com esse tipo de nome de massa. Nossa hipótese é de que os numerais podem se combinar diretamente apenas com nomes massivos que denotam objetos no mundo, como pitf $i$ 'castanha' e rodo 'batata'.

Os dados com os numerais encontrados na literatura e os dados coletados mostram que essa língua não é classificadora, já que nomes contáveis se combinam diretamente com numerais, enquanto nomes de substância precisam de um sintagma de medida. Mostram ainda que essa não é uma língua como o Yudja porque não permite contar diretamente os nomes de substância, contrastando com o exemplo em (8).

Concluímos, então, que o Rikbaktsa apresenta morfologia de plural, com a peculiaridade de ter uma forma plural específica para marcar referentes humanas fêmeas/femininas. O morfema de plural seleciona nomes contáveis, sendo agramatical com nomes de massa substância. Os numerais se combinam diretamente com nomes contáveis e aparentemente também com nomes de massa que denotam objetos no mundo, como rodo batata' e pitfi 'castanha'. Com nomes de massa para substância, é obrigatória a presença de um sintagma de medida. Estas são, portanto, indicações de que se trata de uma língua marcada para número. Mostramos também que essa é uma língua com sintagmas nus.

\section{Um morfema especializado em nomes de massa: $n a$}

Não há dúvidas de que a distinção massivo-contável ocorre nos determinantes em línguas como o inglês e o português: much é massivo, enquanto muitos em português é contável. Em Rikbaktsa, como mostra Silva (2011), há morfologia exclusiva para nomes de massa, que transparece no demonstrativo na 'massivo.proximal', entre outras formas, e é o foco desta seção.

O Kubeo (Tukano-Oriental) apresenta uma propriedade semelhante, mas, diferentemente do Rikbaktsa, parece marcar massa apenas no demonstrativo. Segundo Chacon (2012), a distinção massivo-contável em Kubeo se manifesta, entre outras maneiras, através da flexão dos pronomes demonstrativos. Além de distinguir referentes próximos e distantes, os demonstrativos distinguem nomes animados e inanimados, sendo os últimos classificados entre massivos e contáveis. Os demonstrativos massivos são $i=e$ e $\tilde{a} d \tilde{\imath}=e$, em que $i=$ forma os demonstrativos próximos, $\tilde{a} d \tilde{\imath} \#$ forma os distantes e $=e$ corresponde à marcação de massa.

Notemos ainda que em nenhuma das línguas descritas em Lima \& Rothstein (2020) este tópico aparece. Talvez porque não haja, no questionário, uma seção para os demonstrativos e artigos, ou simplesmente porque esta não é uma propriedade recorrente nas línguas.

Silva (2011) mostra que, em Rikbaktsa, os demonstrativos diferenciam referentes próximo/distante do falante (próximo é não marcado e - $h i$ é o morfema para distante), além de marcarem singular/plural para referentes atômicos (forma feminina e não feminina). Há ainda 
uma forma para nomes massivos: o demonstrativo $n a$, que indica referentes massivos próximos, enquanto a forma $n o-h i,{ }^{13}$ referentes massivos distantes.

Em (24a), o demonstrativo singular ta se combina com um nome singular, enquanto em (24b), o demonstrativo plural fa concorda com o nome, no plural.
a. $\quad t a$
hui
DEM.NF.SG
árvore
'esta é uma árvore'
(Silva 2011:58)
b. $\quad \int a$
hui-t fa
DEM.NF.PL
árvore-NF.PL
'estas são árvores'14

Por outro lado, vemos o demonstrativo massivo $n a$ acompanhando nomes de massa, tanto nomes de substância (24c), quanto de objetos (24d):
c. na tumi
DEM.MAS chicha
'isto é chicha'
d. na rodo
DEM.MAS batata
'isto é batata

(Silva 2011: 58)

Com base nos dados acima, vemos que a oposição massa e contável acontece no demonstrativo: $n a$ versus $t a /$ fa. Segundo Silva (2011), as sequências em (25) são agramaticais:
a. *na hui-t $\quad$ fa
DEM.MAS árvore-NF.PL
b. $\quad * t a \quad$ tumi
DEM.NF.SG chicha
c. *na tumi-t t $a$
DEM.MAS chicha-NF.PL

O interessante é que essa distinção massa e contável reaparece em vários lugares nessa língua. Diferentemente de línguas como o inglês e o português, em Rikbaktsa há um pronome pessoal especializado em nomes de massa. Os pronomes pessoais de terceira pessoa são formados pela combinação da forma $a$ - com as mesmas formas dos demonstrativos: $a$ - $t a$ 'ele', $a$ - $\int a$ 'eles', $a$-na 'ele.massivo'. ${ }^{15}$ A forma $a$-na só retoma nomes de massa.

Em (26), o pronome $a$-ta 'ele' se refere a uma entidade mítica, Põnopõno:

$\begin{array}{llll}\boldsymbol{a}-\boldsymbol{t a} & k t a & k e & b a \\ \text { 3-NF.SG } & \text { FOC.NF.SG } & \text { INFER } & \text { C.ENF } \\ \text { 'Parece que era ele mesmo, } & & \end{array}$

\footnotetext{
${ }^{13}$ A combinação das raízes dos demonstrativos próximos + morfema -hi provoca uma mudança morfofonológica nestas raízes: as vogais /a/da raiz se tornam /o/ diante de - $h i$, assim: na-hi $\rightarrow$ no-hi

${ }^{14}$ Esta língua não tem cópula.

${ }^{15}$ Veja o quadro em Silva (2011:52) para todas as formas pronominais da língua, incluindo a terceira pessoa feminina. Para os nossos propósitos basta o contraste entre contável e massa.
} 
$\boldsymbol{a}-\boldsymbol{t} \boldsymbol{a}=$ to $\quad n i-\phi-a p \dot{\boldsymbol{t}}-\mathrm{k} \boldsymbol{t} k=$ to

3 -NF.SG=ENF 3SUJ-PAS-esperar-CONT=ENF

'ele mesmo que estava esperando'

(Silva 2011: 389)

Em (27), katiktfa 'meus irmãos', um nome contável, como demonstra sua combinação com o morfema de plural -t $\int a$, é retomado pelo pronome plural $a-\int a$ 'eles'.

$k a-t i k-t \int a=j a$

$\boldsymbol{a}-\int \boldsymbol{a}=$ to $\quad \phi-m i-n a-k a$

2POSS.SG-irmão-PL.NF=INTER

$3-\mathrm{NF} . \mathrm{PL}=\mathrm{ENF}$ 1SUJ-NPAS-DIR+AUX-CONT

'e meus irmãos, será que eles ainda vivem, me pergunto? (lit.: eles existem? Eu me pergunto)

(Silva 2011: 229)

Em (28), em resposta à pergunta 'você apagou o fogo?', se referindo ao fogo à lenha, dentro de casa, a falante responde usando o pronome exclusivo para nomes massivos, $a$ - $n a$

$\boldsymbol{a}-\boldsymbol{n a}=$ to

$3-\mathrm{MAS}=\mathrm{ENF}$

'(lit.) (existe) ele mesmo!' 'ainda tem (fogo)!'

Em (29), a-na retoma huak 'pele/casca', um nome de massa (a despeito de aparecer plural na tradução):

$\begin{array}{lll}\text { resho } & h u a k-t i & b o k \sim b o k \\ \text { jenipapo } & \text { pele/casca-ACUS } & \text { RED tirar } \\ \text { 'ela tirou as cascas de jenipapo' } & \end{array}$

'ela tirou as cascas de jenipapo'

$\begin{array}{llll}\text { iware } & \boldsymbol{a}-\boldsymbol{n} \boldsymbol{a}=t \boldsymbol{t} & b o k \sim b o k & n i-\phi-a=t a t f a=h i \\ \text { depois } & 3-\mathrm{MAS}=\mathrm{ACUS} & \text { RED } \sim \text { tirar } & \text { SUJ-PAS-AUX=F.SG=? }\end{array}$

'depois, a que as tirou (as cascas de jenipapo) disse (...)'

(Silva 2011: 53)

A forma para os nomes massivos, $n a$, também aparece em algumas proformas interrogativas morfologicamente complexas, compostas pela interrogação $h \tilde{a}$, acompanhada de clíticos que, segundo Silva (2011), são resultantes da gramaticalização dos demonstrativos já apresentados. Aqui, a mesma oposição aparece entre as formas contáveis e a forma massiva. Para os referentes não femininos temos $h \tilde{a}=t a$ 'para/onde' com nomes no singular, e $h \tilde{a}=t f a$ 'para onde' com nomes plurais. Em (30) e (31) temos a forma interrogativa singular com nome contável não feminino, foksrotfapo 'faquinha', e com nome feminino, aje 'sua mãe', respectivamente:

\begin{tabular}{|c|c|}
\hline$h \tilde{a}=t a$ & foksro-tfapo \\
\hline INTER $=$ NF.SG & cutia-dente \\
\hline
\end{tabular}

'onde está a faquinha?'

(Silva 2011: 216)

\begin{tabular}{|c|c|c|}
\hline$h \tilde{a}=t a t \int a$ & Jkaraba & $a$-je \\
\hline $\begin{array}{l}\text { INTER=F.SG } \\
\text { onde está su }\end{array}$ & INTER & $\begin{array}{l}\text { 2POSS.SG-mã } \\
\text { (Silva 2011: 217) }\end{array}$ \\
\hline
\end{tabular}

Se comparamos (30) e (32), vemos as formas interrogativas hãata e hã $t$ t $a$ interrogando, respectivamente, sobre o lugar em que está a faquinha e o lugar em que estão os peixes. Em (32) há concordância com o nome, marcado com o morfema de plural. 


$$
\begin{array}{ll}
\boldsymbol{h} \tilde{\boldsymbol{a}}=\boldsymbol{t} \boldsymbol{d} \boldsymbol{a} & \text { piknu-t } \int a \\
\text { INTER=NF.PL } & \text { peixe-NF.PL } \\
\text { 'onde está/tem peixes?' }
\end{array}
$$

Se o nome é massivo, a forma interrogativa irá se combinar com o demonstrativo para massa, resultando em $h \tilde{a}=n a$. Em (33), a forma $h \tilde{a}=n a$, por sua vez, interroga sobre o nome de massa pitfi 'castanha':

$\begin{array}{lc}\boldsymbol{h} \tilde{\boldsymbol{a}}=\boldsymbol{n} \boldsymbol{a} & \text { pitf } i \\ \text { INTER=MAS } & \text { castanha } \\ \text { 'onde está/tem castanha? }\end{array}$

(Silva 2011: 217)

\section{na pitfi \\ DEM.MAS castanha \\ 'aqui tem castanha', 'estas são castanha'}

A marca para massa aparece ainda em construções que Silva (2011) nomeia de atributivas. Estas construções merecem um estudo à parte e neste artigo, apresentamos uma descrição simplificada. As atributivas são construções formadas por nomes resultados de nominalização de uma raiz verbal, combinada a um elemento que a autora denomina predicativizador, $\tilde{i}$-, ${ }^{16}$ ao qual se agregam clíticos que são resultantes da gramaticalização dos demonstrativos e das marcas de gênero e número (Silva 2011: 312). Para os nossos propósitos, interessa notar o padrão recorrente: de um lado, as formas contáveis (femininas e não femininas), e de outro, a forma massiva.

Em (34), o clítico t fa, que, como dito acima, tem origem nas marcas de gênero e número, acompanha o predicativo ako 'ser alegre'. Ele indica que o referente é contável. Oka 'neto', aparece com o plural, concordando, portanto, com o predicativo.

$$
\begin{array}{ll}
\text { ka-fte-oka-t } \boldsymbol{f a} & \int-a k o-\tilde{\imath}=\boldsymbol{t} \boldsymbol{f a} \\
\text { 1POSS.SG-filha-filho-NF.PL } & \text { 3PL-ser.alegre-EST.AFIR=NF.PL } \\
\text { 'os meus netos são alegres' } &
\end{array}
$$

O mesmo padrão ocorre em (35), a marca de massa na acompanha o predicativo, indicando que o referente deste é massivo, raro 'mingau':

$$
\begin{array}{ll}
\text { mi-raro } & \text { t } \int \hat{i}-\int a p a-\tilde{\imath}=\boldsymbol{n a} \\
\text { 1POSS.PL-mingau } & \text { 3sG-ser.bonito/bom-EST.AFIR=MAS } \\
\text { 'nosso mingau está bom' }
\end{array}
$$

O papel gramatical desempenhado pela forma na permitiu detectar a presença de um nome flexível em nosso corpus, naha 'ornamentária/colar/joia'. Como já dissemos, esse tipo de nome se caracteriza pela possibilidade de ser utilizado como contável ou massivo. O nome encontrado, naha 'ornamentária/colar/joia', tem uma contraparte contável que admite a combinação com o sufixo de plural $-t \int a$, como em (36), retomado no verbo pelo índice de pessoa plural, $-\int i$ - '3OBJ.PL':

\footnotetext{
${ }^{16}$ Silva (2011: 320) afirma que nesse contexto, a distinção massivo-contável ocorre apenas com as construções atributivas afirmativas, ou seja, formadas pelo predicativizador $\tilde{l}^{-}$'estativo afirmativo', que se opõe ao morfema bii- 'estativo negativo'. Essa questão precisa ser investigada.
} 


$$
\begin{array}{lll}
\text { ikra } & \text { ka-naha-tfa } & \phi \text {-mi-fi-wo wo } \\
\text { eu } & \text { 1POSS.SG-colar-NF.PL } & \text { 1SUJ-NPAS-3OBJ.PL-RED furar } \\
\text { 'eu vou furar meus colares' (furar as sementes para fazer meus colares) }
\end{array}
$$

No entanto, o dado em (37) mostra que naha também pode funcionar como um nome massivo, nesse caso não se combina com o sufixo de plural $-t \int a$, e é retomado pelo clítico $=n a$

\begin{tabular}{|c|c|}
\hline mi-naha & $t / i-\int a p a-\tilde{\imath}=\boldsymbol{n a}$ \\
\hline 1POSS.PL-colar & 3SG-ser.bonito/bom-EST.AFIR=MAS \\
\hline
\end{tabular}
no predicativo:

A tradução de (37) não faz jus ao que está sendo expresso, melhor seria traduzir por: 'nossa ornamentária é bonita'. A forma naha é, portanto, um nome de massa e um nome contável, o que permite concluir que essa língua tem nomes flexíveis.

\section{Conclusão}

O artigo oferece uma descrição da distinção massivo-contável na gramática Rikbaktsa com o objetivo de contribuir para os estudos sobre a gramática através das línguas, mas também construir um conhecimento sobre essa língua que possa ajudar na sua valorização e vitalização. O fundo teórico é a discussão atual sobre os nomes nas línguas naturais, em particular os parâmetros semânticos de Chierchia $(2010,2015$, no prelo) e na literatura recente sobre o tema (Kiss et al. no prelo; Rothstein 2010, 2017, no prelo). Nosso ponto de partida foi o questionário de Lima \& Rothstein (2020) para as línguas indígenas brasileiras.

Investigamos a distinção massivo-contável na literatura sobre a língua Rikbaktsa (Boswood 1978, 1971; SIL 2007; Silva, 2011), e também em narrativas espontâneas coletadas e transcritas por Silva durante trabalho de campo entre os anos de 2003 a 2010 (muitas delas inéditas), além de uma elicitação de dados via internet. A análise proposta permitiu construir hipóteses que poderão ser aperfeiçoadas com uma coleta controlada de dados junto a falantes nativos.

Comparando os nossos resultados com aqueles descritos para as línguas indígenas analisadas em Lima \& Rothstein (2010), é possível afirmar que o Rikbaktsa é uma língua que tem nomes nus e a morfologia de plural distingue massa e contável, já que nomes de substância não aceitam morfologia de plural. Esta não é obrigatória no nome, embora nos índices pessoais atachados aos verbos seja obrigatória a especificação do número. Em Rikbaktsa, o plural ocorre com nomes contáveis, sem outras restrições. Além disso, há formas de plural específicas para fêmeas humanas.

A pesquisa sobre os numerais mostra indícios de que é possível contar massa diretamente, se o nome massivo denotar objetos no mundo. Nossa hipótese é que nomes massivos que denotam objetos se combinam diretamente com numerais, enquanto nomes de substância exigem um sintagma de medida. Nesse sentido, nossa pesquisa corrobora a generalização feita por Lima \& Rothstein (2020) de que marcação de plural e contabilidade são conceitos independentes. Identificamos um exemplo de nome de massa falso e um exemplo de nome flexível. Como já notamos, essas são propriedades de línguas marcadas para número, de acordo com os parâmetros de Chierchia (2010). Trata-se, portanto, de uma língua que marca número, mas diferentemente de outras línguas desse tipo, como o inglês, há indícios de que a marcação de plural não é obrigatória no nome.

Em Lima \& Rothstein (2020) não há menção de línguas que exibem um morfema exclusivo para expressar massa. Isso pode ser apenas uma consequência da estrutura do 
questionário, que não verifica o comportamento de demonstrativos e pronomes, ou podemos estar aqui efetivamente diante de uma língua com uma propriedade incomum. Chacon (2012) mostra que Kubeo (Tukano-Oriental) também marca massa morfologicamente. Em Rikbaktsa, o demonstrativo $n a$, bastante produtivo na língua, só pode se combinar com nomes massivos. Essa morfologia para massa aparece também no pronome pessoal com referente massivo, $a-n a$ 'elemassivo'; na proforma interrogativa, $h \tilde{a}=n a$ 'onde massivo'; e em predicativos, com o predicativizador $\tilde{l}-$, quando está combinado com um nome massivo. Esses são indícios de que o contraste massivo e contável é obrigatório no determinante e nas formas pronominais, embora o plural seja facultativo no nome.

Além de contribuir para a pesquisa sobre a semântica dos sintagmas nominais através das línguas, essa investigação permite subsidiar diferentes atividades de coleta para serem realizadas em campo com o objetivo de aprofundar o tema aqui tratado. Sem dúvida, ela contribui ainda para a documentação, salvaguarda e valorização da língua e da cultura Rikbaktsa.

\section{$\overline{\text { Referências }}$}

Áthila, Adriana (2006). "Arriscando corpos": Permeabilidade, alteridade e as formas da socialidade entre os Rikbaktsa (Macro-Jê) do sudoeste amazônico (Tese de doutorado em Sociologia e Antropologia). Rio de Janeiro: Universidade Federal do Rio de Janeiro, Programa de Pós Graduação em Sociologia e Antropologia.

Áthila, Adriana (2019). A "Caixa de Pandora". Representação, diferença e tecnologias nativas de reprodução entre os Rikbaktsa (Macro-Jê) do Sudeste Amazônico. Revista Antropologia 62(3): 710-743. https://doi.org/10.11606/2179-0892.ra.2019.165225

Barner, David; Snedeker, Jesse (2005). Quantity judgments and individuation: Evidence that mass nouns count. Cognition 97(1): 41-66. https://doi.org/10.1016/j.cognition.2004.06.009

Boswood, Joan (1971). Phonology and morphology of Rikbaktsa and a tentative comparison with languages of the Tupi and Jê families (MA thesis). Reading University.

Boswood, Joan. (1978 [2007]). Quer falar a língua dos canoeiros? Rikbaktsa em 26 lições. Brasília: Summer Institute of Linguistics.

https://www.sil.org/system/files/reapdata/33/65/14/336514431674588558668664356625639224414/RK Gram.pdf

Chacon, Thiago Costa (2012). The phonology and morphology of Kubeo: The documentation, theory, and description of an Amazonian language (Ph.D. dissertation). University of Hawai'i: http://etnolinguistica.wdfiles.com/local--files/tese\%3Achacon-012/chacon_2012_kubeo.pdf

Chierchia, Gennaro (1998). Plurality of mass nouns and the notion of 'semantic parameter'. Events in Grammar. In Susan Rothstein (ed.), Events and grammar, pp. 53-103.Kluwer Academic Publishers.

Chierchia, Gennaro (2010). Mass nouns, vagueness and semantic variation. Synthese 174: 99-149. https://doi.org/10.1007/s11229-009-9686-6

Chierchia, Gennaro (2015). How universal is the mass/count distinction? Three grammars of counting. In Audrey Li; Andrew Simpson; Wei-Tien Dylan Tsai (eds.), Chinese syntax in a cross-linguistic perspective, pp. 147-176. Oxford University Press. 10.1093/acprof:oso/9780199945658.003.0006

Chierchia, Gennaro (no prelo). Mass vs. Count: Where do we stand? Outline of a theory of semantic variation. In T. Kiss; F.J. Pelletier; H. Husic (eds.), Things and Stuff: The Semantics of the Count-Mass Distinction [Chapter 2]. Cambridge, UK: Cambridge University Press. 
Frazier, L; Frisson, S. (2005). Carving up word meaning: Portioning and grinding. Journal of Memory and Language 53(2): 277-291. https://doi.org/10.1016/j.jml.2005.03.004

Galucio, Ana Vilacy; Costa, Carla. Nascimento (2020). Count-mass distinction in Sakurabiat. Linguistic Variation 20(2): 336-351. https://doi.org/10.1075/lv.00025.gal

Kiss, Tibor; Pelletier, Francis J.; Husic, Halima (no prelo). Things and stuff: The semantics of the count-mass distinction. Cambridge, UK: Cambridge University Press.

Lima, Suzi (2010). About the count-mass distinction in Yudja: A description. In Beth Rogers; Anita Szkay (eds.), The Fifteenth Workshop on Structure and Constituency in Languages of the America, vol. 29: 157-164. Vancouver: University of British Columbia.

Lima, Suzi (2014). The acquisition of the count/mass distinction in Yudja (Tupi): Quantifying 'Quantity' and 'Number'. In Chia-Ying Chu et al. (eds.), Selected Proceedings of the 5th Conference on Generative Approaches to Language Acquisition North America (GALANA 2012), pp. 181-190. Somerville, MA: Cascadilla Proceedings Project. http://www.lingref.com/cpp/galana/5/paper3079.pdf

Lima, Suzi; Rothstein, Susan (2020). A typology of the mass/count distinction in Brazil and its relevance for mass/count theories. Linguistic Variation 20(2): 174-218. https://doi.org/10.1075/lv.20.2

Moltmann, Friederike (ed.) (2020). Mass and count in linguistics, philosophy and cognitive science. Amsterdam: John Benjamins. https://doi.org/10.1075/lfab.16

Pacini, Aloir (1999). Pacificar: Relações interétnicas e territorialização dos Rikbaktsa (Dissertação de mestrado). Universidade Federal do Rio de Janeiro. https://comin.org.br/wp-content/uploads/2019/08/relacoes-interetnicas-1282915485.pdf

Pelletier, Francis J. (1975). Non-singular reference: Some preliminaries. Philosophia 5(4): 451-465. https://doi.org/10.1007/978-1-4020-4110-5_1

Pelletier, Francis Jeffry (2009). Kinds, things and stuff: Mass terms and generics. Oxford: Oxford University Press. https://doi.org/10.1093/acprof:oso/9780195382891.001.0001

Pelletier, Francis Jeffry (2012). Lexical nouns as both +mass and +count, and neither +mass nor +count. In Massam, Diane (ed.), Count and mass across languages, pp. 1-25. Oxford: Oxford Press. https://doir.org/10.1093/acprof:oso/9780199654277.003.0002

Pires de Oliveira, Roberta; Rothstein, Susan (2011). Bare singular noun phrases are mass in Brazilian Portuguese. Lingua 121(15): 2153-2175. https:// doi.org/10.1016/j.lingua.2011.09.004

Polegatti, Geraldo Aparecido (2013). A matemática Rikbaktsa para o povo Rikbaktsa: Um olhar da etnomatemática na Educação Escolar Indígena (Dissertação de mestrado em ciências). Seropédica: Universidade Federal Rural do Rio de Janeiro, Instituto de Agronomia. https://tede.ufrrj.br/jspui/handle/jspui/3568

Rodrigues, Aryon Dall'igna (1986). Línguas Brasileiras: Para o conhecimento das línguas indígenas. São Paulo: Edições Loyola.

Rothstein, Susan (2010). Counting and mass/count distinction. Journal of Semantics 27(3): 343-397. http://semantics.uchicago.edu/kennedy/classes/f11/na/docs/rothstein10.pdf http://doi.org/10.1093/jos/ffq007

Rothstein, Susan (2017). Semantics for counting and measuring. Cambridge University Press. https://doi.org/10.1017/9780511734830

Rothstein, Susan; Pires de Oliveira, Roberta (2020). Comparatives in Brazilian Portuguese: counting and measuring. In Moltmann, Friederike (ed.), Mass and count in linguistics, philosophy, and cognitive science [Language Faculty and Beyond 16], pp. 141-157. https://doi.org/10.1075/lfab.16.07rot 
Rothstein, Susan (no prelo). Counting, plurality, and portions. In T. Kiss, F.J. Pelletier, and H. Husic (eds.), Things and stuff: The semantics of the count-mass distinction. [Chapter 3]. Cambridge, UK: Cambridge University Press.

SIL (2007). Dicionário Rikbaktsa/Português e Português/Rikbaktsa. Associação Internacional de Linguística, Cuiabá: MT.

https://www.sil.org/system/files/reapdata/23/74/77/23747713988086297350702187859467170352/RKDic.pdf

Silva, Léia de Jesus (2011). Morphosyntaxe du Rikbaktsa (Amazonie brésilienne) (Tese de doutorado). Curso de letras. Université Denis Diderot, Paris.

http://www.etnolinguistica.org/local--files/tese:silva-2011b/silva_2011_rikbaktsa.pdf

Soja, Nancy N.; Carey, Susan; Spelke, Elizabeth S. (1991). Ontological categories guide young children's inductions of word meanings: Object terms and substance terms. Cognition 38(2): 179-211. https://doi.org/10.1016/0010-0277(91)90051-5

ABREVIATURAS

$\begin{array}{llll}1 & \text { primeira pessoa } & \text { INFER } & \text { inferencial } \\ 2 & \text { segunda pessoa } & \text { INTER } & \text { interrogativo } \\ 3 & \text { terceira pessoa } & \text { MAS } & \text { massivo } \\ \text { ACUS } & \text { acusativo } & \text { NEG } & \text { negação } \\ \text { ALAT } & \text { alativo } & \text { NF } & \text { não fêmea } \\ \text { AUX } & \text { auxiliar } & \text { NPAS } & \text { não passado } \\ \text { C.ENF } & \text { certeza enfática } & \text { OBJ } & \text { objeto } \\ \text { CL } & \text { classificador } & \text { PAS } & \text { passado } \\ \text { CONT } & \text { continuativo } & \text { PL } & \text { plural } \\ \text { COR } & \text { correferencial } & \text { POSS } & \text { possessivo } \\ \text { DEM } & \text { demonstrativo } & \text { RED } & \text { reduplicação } \\ \text { DIM } & \text { diminutivo } & \text { RESTR } & \text { restritivo } \\ \text { ENF } & \text { ênfase } & \text { SG } & \text { singular } \\ \text { EST.AFIR } & \text { estativo afirmativo } & \text { SIM } & \text { similitivo } \\ \text { F } & \text { fêmea } & \text { SUJ } & \text { sujeito } \\ \text { FOC } & \text { focalização } & & \end{array}$

Recebido: 21/9/2020

Versão revista 1: 28/2/2021

Versão revista 2: 19/4/2021

Versão revista 3: 16/5/2021

Aceito: $25 / 5 / 2021$

Publicado: $31 / 5 / 2021$ 